Cahiers de recherches médiévales

Journal of medieval studies

4 | 1997

Être père à la fin du Moyen Âge

\title{
Images du père de famille au Moyen Âge
}

\section{Danièle Alexandre-Bidon}

\section{OpenEdition}

Journals

Édition électronique

URL : https://journals.openedition.org/crm/963

DOI : $10.4000 / \mathrm{crm} .963$

ISSN : 1955-2424

Éditeur

Honoré Champion

Édition imprimée

Date de publication : 15 décembre 1997

ISSN : 1272-9752

\section{Référence électronique}

Danièle Alexandre-Bidon, «Images du père de famille au Moyen Âge », Cahiers de recherches

médiévales [En ligne], 4 | 1997, mis en ligne le 04 septembre 2007, consulté le 15 décembre 2022

URL : http://journals.openedition.org/crm/963 ; DOI : https://doi.org/10.4000/crm.963

Ce document a été généré automatiquement le 15 décembre 2022.

Tous droits réservés 


\title{
Images du père de famille au Moyen Âge
}

\author{
Danièle Alexandre-Bidon
}

Le contexte historiographique

Pour Philippe et Émeline

1 Nul ne songerait plus aujourd'hui à prétendre que les pères n'ont aucun rôle à jouer dans l'éducation et la vie affective des enfants. Et pourtant, depuis les historiens ou érudits du XIX ${ }^{\mathrm{e}}$ siècle, puis les historien(nes) féministes des années 60 et suivantes, les pères médiévaux n'ont guère eu droit à l'attention des spécialistes de l'histoire de l'enfance. L'Histoire des mères, d'Yvonne Knibielher et Catherine Fouquet, publiée en 1977, éliminait par nécessité toute référence aux pères. L'Histoire de la famille, publiée en 1986, a bien consacré quelques pages au thème "Pères et fils ", mais les seuls exemples de relations affectives présentés étaient soigneusement (?) choisis dans le domaine du négatif : le célèbre refus de Philippe le Bon, duc de Bourgogne d'assister au baptême de sa petite-fille Marie; l'interprétation quasi systématique des morts accidentelles par étouffement ou brûlure dans les flammes du foyer ou l'eau bouillante d'une marmite comme infanticides déguisés; l'inceste, dit à juste titre violemment réprimé ; l'exclusion $\mathrm{du}$ fils lors $\mathrm{du}$ remariage du père; l'absence de représentations iconographiques de cet âge de la vie qu'est l'enfance. La relation privilégiée du père à l'adolescent mâle y était cependant soulignée mais un jugement accablant affirmait pour finir " un niveau faible de la relation père-fils... », " des rapports difficiles entre un père vieilli et un fils trop jeune ", pour conclure que «le père est une figure lointaine, alors que la mère, très présente, assure l'influence féminine sur le fils ${ }^{1}{ }^{1} .$.

2 L'Histoire des femmes, en 1991, ne pouvait, de par sa thématique générale même, consacrer qu'un faible nombre de pages au rôle de l'homme dans le foyer; mais force est de constater que les auteurs y suivent assez fidèlement, trop sans doute, les textes normatifs médiévaux sur la famille sans tenir compte des sources plus "familières", où l'on aurait pu découvrir l'intimité père-enfant; ainsi, si "diriger la famille» est au programme, les relations affectives entre pères et enfants sont définies plutôt négativement: les mots de saint Thomas - la mère aime son enfant plus que le père - 
sont cités en premier; si les enfants, selon Jacques de Voragine, tendent malgré tout à aimer davantage leur père que leur mère, ce n'est, pour Thomas, que par devoir. Les pères seraient «surtout responsables de l'éducation des garçons une fois ces derniers sortis de l'enfance $»^{2}$.

Cependant, à n'associer, somme toute de manière très conventionnelle, que les femmes aux jeunes enfants, pour réserver aux hommes la seule responsabilité de l'adolescence, l'histoire a perdu de vue que les pères aussi jouaient un rôle dans le vécu des toutpetits. Ce besoin de n'associer que la mère au tout-petit et le père à l'adolescent, encore toute empreint de l'esprit bourgeois du siècle dernier et de la première moitié du nôtre, n'a donc pas disparu de nos mentalités revues et corrigées par la libération des femmes. De fait, l'histoire des femmes et des enfants a longtemps été revendiquée par des historiennes... qui, plus ou moins inconsciemment, et souvent plus que moins, ont eu tendance à exclure la mâle part du Moyen Âge et à focaliser sur la femme, qu'il était alors à la mode de promouvoir avec passion. Il a par conséquent longtemps été de rigueur, pour ne pas dire "politiquement correct", de ne pas associer intimement la petite enfance et la paternité. Grave erreur: il a manqué aux études sur l'environnement affectif de l'enfance une dimension sans doute tout aussi essentielle que celle qui réunit mère et enfant. Une telle attitude (générale) est assurément influencée par nos mentalités contemporaines. Dans la société actuelle, les "nouveaux pères" d'après 1968 ne semblent pas avoir laissé de descendance; les hommes, laissés perplexes devant leurs nouveaux droits et devoirs, n'ont guère été encouragés à rompre l'habitude soi-disant ancestrale consistant à laisser aux mères les soins des tout-petits... Le phénomène est aujourd'hui jugé assez grave pour avoir suscité une journée d'études et de réflexion de la Fondation pour l'enfance 3 .

4 En 1990, un ouvrage sur les relations père-fils au XVIII ${ }^{e}$ siècle ${ }^{4}$ mettait en valeur, tentation propre à tous les modernistes ${ }^{5}$, l'évolution du sentiment paternel aux temps modernes seulement : «le nouveau rôle de père, axé sur l'indulgence et la bienveillance, qui se met lentement en place, n'est pas de leur âge. $»^{6}\left[\mathrm{~d}^{\prime}\right.$ hommes du XVII ${ }^{\mathrm{e}}$ siècle]. L'auteur ne le dit pas, mais le lecteur a du mal à se dire que tout n'allait pas pis encore au Moyen Âge... Dans ce contexte, l'Histoire des Pères et de la paternité, publiée la même année par Jean Delumeau et Daniel Roche, est apparue fort originale et novatrice, tout en ne consacrant, malheureusement, aux géniteurs médiévaux qu'une portion congrue. Ils y apparaissent surtout dans la fonction hélas conventionnelle pour ne pas dire intemporelle du "père fouettard". On y lit, pour l'époque médiévale, qu' "à vrai dire le père parait assez peu $»^{7}$. L'homme, et le père en particulier, a longtemps eu mauvaise presse. À l'exception de Pierre Riché, qui pour le haut Moyen Âge s'est fait le chantre de la famille, de l'enfance et de l'éducation, il faut admettre que les historiens du sexe fort n'étaient guère tentés par ce type de sujet - et que pour leur part les femmes n'étaient guère enclines à étudier les pères... En 1994-1995, lors d'une exposition de la Bibliothèque nationale placée sous le double commissariat de Pierre Riché et de moimême, nous avons tenté de remettre le père de famille à l'honneur grâce à des enluminures de manuscrits et une statue éclairantes dans ses rapports à la petite enfance ${ }^{8}$.

Il faut aujourd'hui de jeunes historiens médiévistes pères de famille et spécialistes de l'histoire de l'enfance, chose rare, pour qu'enfin on cherche à mieux cerner, sans les cantonner aux seuls et anciens stéréotypes du père sévère - et éducateur, mais de loin les rôles familiers du pater familias. Parler du père est redevenu, enfin, de mise car les 
sources nouvelles éclairant les rôles paternels (récits de miracles, lettres de rémission, iconographie) sont désormais connues de tous et que la phase sans doute inévitable de militantisme historique paraît bel et bien achevée. On sait maintenant que les pères médiévaux apprenaient à marcher à leurs enfants d'un an et demi, prenaient la température tous les jours de leurs enfants malades baignaient les enfants ${ }^{9}$, ou surveillaient leurs jeux en les consolant et en les défendant lorsqu'un autre gamin leur avait dérobé leurs jouets ${ }^{10}$, voire encore achetaient la layette, comme le noble Paston ${ }^{11}$. Les pères "maternent" au Moyen Âge, à l'image de Joseph ou, plutôt, si le Joseph des images s'occupe tendrement de l'Enfant, c'est sans doute à l'image des vrais pères qu'il le fait.

L'image du père

6 La même réticence à montrer des pères aux gestes maternels s'est longtemps affirmée lorsqu'il s'est agi de donner à voir des images du père. Tout au long des XIX ${ }^{\mathrm{e}}$ et $\mathrm{XX}^{\mathrm{e}}$ siècles, les seuls pères "licites" étaient des pères dominateurs, non des pères tendres, qui existent cependant, et en masse non négligeable, dans l'iconographie médiévale, qu'il s'agisse de Joseph ou des pères ordinaires. Cette image a mis du temps à percer et aujourd'hui encore on ne la donne à voir qu'exceptionnellement. Les livres d'art, riches en images de la Sainte Famille, ont toujours et systématiquement privilégié la double vision d'un Joseph soit jaloux de cet Enfant qui n'était pas de lui, soit indifférent à son entrée dans sa famille; il se chauffe les pieds au-dessus d'un brasero, dédaignant les Mages qui viennent pour adorer l'Enfant, ou dort dans son coin tandis que Marie s'occupe du bébé... Au mieux il éclaire d'une bougie la scène où Marie prie à genoux en adoration devant le nourrisson. Les mêmes livres d'art, méprisant, comme il a longtemps été de rigueur, la banalité de la vie quotidienne au profit des motifs esthétiques et religieux purs et durs, n'ont pour ainsi dire jamais non plus laissé la parole aux "vrais" pères, ceux des milieux sociaux les plus divers et qui, des nobles aux paysans ou aux pauvres mendiants et faux pèlerins vagabondant en famille de lieu d'accueil en hôpital, s'occupent réellement de leurs petits enfants...

7 Et pourtant, de telles images existent et bien d'autres représentations de Joseph en père de famille que celles du cocu jaloux et furieux, indifférent au mieux, surgissent du corpus immense des enluminures médiévales. On en a vues à l'occasion, au fil de quelque article, ainsi en 1976 grâce à l'abbé Garnier dans un numéro spécial de la revue Archeologia consacrée à la peinture de manuscrit ${ }^{12}$ : la première illustration y renvoyait au thème de Joseph cuisant le "papet » ou "papa » (i. e. la bouillie) pour son petit Jésus. Mais, en vis-à-vis et en caractères gras, un commentaire en sous-titre de l'article tendait à laisser supposer l'extrême rareté de telles illustrations en précisant : «Nous présentons ici un florilège - qui a été difficile à établir...». De quoi décourager la recherche en matière d'iconographie! Aussi de telles images sont-elles restées au désert; dans les années 80 , seules les mères ont eu la faveur des images. Encore dans les années 90, pas l'ombre d'un père avant le XVIII ${ }^{e}$ siècle dans Le Peintre et l'enfant, de $\mathrm{D}$. Spiess. Les images de tendresse paternelle apparaissent à peine même dans les ouvrages pourtant dédiés au rôle des pères : dans l'Histoire des pères et de la paternité, les images médiévales du père étaient pour la plupart rares - et ambiguës : c'est Joseph qui, assis dans son coin et tournant le dos à l'Enfant, retaille ses chausses usées par les chemins ; c'est Noë en père ivre mort et rendu ridicule par sa nudité exhibée dont son fils Cham se gausse; c'est tout de même Joseph qui tend au feu le lange de son bébé ; mais on persiste à affirmer, dans une perspective ariésienne lointaine, que «quant au 
nouveau-né, avant la seconde moitié du XVIII siècle, il n'est pas sur les images le tendre objet des soins du père, sauf lorsque l'enfant est Jésus et le père saint Joseph $»^{13}$ sans se demander si d'autres sources ne complètaient pas les informations des (trop rares) images connues des historiens.

Lorsque nous-mêmes, dans un premier essai encore estudiantin, L'Enfant à l'ombre des cathédrales $^{14}$, avons travaillé sur la puériculture, c'est justement l'image, et notamment l'enluminure des manuscrits gothiques, alors peu connue et sous-représentée, qui nous avait permis de percevoir que les fonctions paternelles ne se limitaient pas aux rôles socio-économiques et à la rigueur d'une éducation donnée aux grands enfants. Même si nous avions choisi d'étudier "le rôle de la mère " avant "le rôle du père " $^{15}$, nous pensions déjà qu'il était nécessaire de mettre en valeur le caractère affectif des relations liant père et enfants, en insistant sur son rôle actif dans le domaine de la puériculture, sa disponibilité envers les jeux des tout-petits : selon les sources et les modèles, on voit le père manier le hochet, fabriquer des jouets, prendre l'enfant dans ses bras, le porter sur son dos, le nourrir, s'attendrir devant les mots d'enfants... Parmi le corpus d'images publiées, nous avions choisi de montrer le Joseph d'une Légende dorée apportant du bois pour le feu devant lequel est baigné l'Enfant Jésus et lui tendant les bras avec amour ${ }^{16}$, ou réchauffant son lange à la flamme ${ }^{17}$. Nous étions conscientes que l'image n'était d'ailleurs pas l'unique moyen d'assurer le lecteur de l'intimité des relations père-enfants: les pères médiévaux écrivaient même des berceuses en "langage bébé", tel le prince poète Charles d'Orléans... ${ }^{18}$

9 À cette exception près, nous connaissions moins bien les pères au quotidien, notamment dans l'iconographie. Seule l'illutration sarcastique des XV joies de mariage ${ }^{19}$, une gravure d'un père exploité par son épouse, l'épée au côté mais le fuseau (symbole féminin) planté dans son bonnet, surchargé du berceau, de la poêle à bouillie des petits, dont l'un réclame sa pitance, un autre accroché à ses basques, soulignait le rôle paternel dans la vie quotidienne des bébés mais une nouvelle fois de manière ambiguë, celle de la revanche des femmes sur les hommes... Depuis dix ans, le dépouillement de centaines, voire de milliers de manuscrits supplémentaires, m'a permis de mieux sérier la relation père-enfants telle que les artistes des $\mathrm{XIII}^{\mathrm{e}}-\mathrm{XV}^{\mathrm{e}}$ siècles, qui étaient euxmêmes laïcs et pères de famille, ont cherché à la représenter. Parallèlement, des sources écrites jusqu'alors peu exploitées, étudiées par d'autres, ont enfin et surtout laissé apparaitre les rôles maternants du père dans la famille médiévale, confortant les intuitions antérieures suscitées par l'étude d'images en nombre encore trop peu fourni. C'est le but de cet article que de faire l'inventaire (toujours incomplet, le corpus d'images enluminées, immense, étant toujours ouvert) des images du père, thème qui pourrait faire l'objet d'une maîtrise ou d'une thèse tant abondent les représentations.

Les sources iconographiques

10 Si l'image de Joseph, à laquelle on s'intéresse de nouveau depuis peu, est assurément un bon révélateur des comportements paternels médiévaux, qu'en est-il de celle des "vrais" pères, ceux qui, issus de tous milieux sociaux, sont mis en scène dans les enluminures? L'image en est plurielle. Elle traduit quasi systématiquement de leur part une relation de proximité affective à ses enfants et un intérêt pour leur sort. Mais l'image du père varie bien sûr selon le type de texte qu'elle illustre. Commençons par le commencement, la Bible: la première impression est globalement négative. Tandis qu'Adam s'épuise à bêcher son champ, par force indifférent à tout ce qui l'entoure, ses deux petits garçons, frères ennemis dès l'enfance, luttent corps à corps sous ses yeux : 
c'est déjà la discorde au sein de la famille. Abraham ensuite n'hésite pas à sacrifier son fils en l'empoignant par les cheveux. L'amour divin est plus fort que l'amour paternel, paradoxe de la civilisation chrétienne qui protège pourtant les petits enfants... Le fils d'Agar est chassé par son père. Passons, ce n'est pas là une image médiévale du père, mais l'illustration d'un texte antique, même s'il est parfois revu et corrigé par la sensibilité médiévale. Dans le nouveau Testament, Dieu lui-même, le père par excellence, fait preuve d'une rare cruauté lorsqu'il laisse son fils aller au supplice et, plus encore, lorsqu'il lui serre lui-même la vis, stricto sensu, pour le vider de son sang sous la croix dans le thème étonnant du "Pressoir mystique"20. Mais Dieu n'est pas un père ordinaire, tant s'en faut...

11 Les encyclopédies, en revanche, devraient être davantage en prise avec les réalités sociales et familiales du Moyen Âge; celle de Barthélemy l'Anglais notamment, écrite au XIII siècle, toujours en usage au $\mathrm{XV}^{\mathrm{e}}$ et même au-delà, presque toujours illustrée, comporte nombre d'images du père. Mais elles font uniquement écho au texte et aux théories médicales de l'époque; quand les hommes figurés aux différents âges de la vie ne sont pas simplement alignés, sans contact particulier des uns aux autres ni des adultes aux enfants, la seule association physique qui se noue s'effectue entre le plus jeune et le plus vieux d'entre eux: la littérature médicale nous dit en effet que leurs constitutions plus fragiles rapprochent ces deux âges de la vie... De telles images masquent ainsi tout rapprochement père-fils, d'une part par le transfert de réalités familiales au profit d'une association théorique enfant-vieillard dans le motif des âges de la vie ${ }^{21}$ - ainsi, au chapitre «De la brièveté de la jeunesse et de la misère de l'âge », un petit garçon soutient un vieillard cassé en deux par les rhumatismes et réduit à sa taille ${ }^{22}$ - d'autre part par l'association père-mère privilégiée aux dépens de l'association parents-enfants: c'est ainsi que l'homme et la femme se tiennent tendrement enlacés tandis que l'enfant joue à leurs côtés, sans que nul ne lui prête attention ${ }^{23}$; qu'on n'en déduise pas pour autant que l'amour entre époux l'emportait forcément sur l'amour parental ou, pis, que les enfants étaient dédaignés des parents : c'est aussi et surtout que logiquement le couple vient avant la procréation... Les images encyclopédiques, hélas, ne sont pas des photos de la vie familiale mais plus souvent des images classificatrices $^{24}$, l'illustration symbolique de théories savantes complexes et pourtant condensées en une seule image.

12 En revanche, d'autres textes normatifs contemporains de telles encyclopédies n'omettent pas, dans leur illustration, de présenter dans une relation affective positive les pères et leurs enfants. C'est notamment le cas dans une traduction de la Politique d'Aristote, aux armes de la famille du Fou ${ }^{25}$, qui est ornée de scènes familiales montrant comment le père doit aimer ses enfants et comment il doit les éduquer personnellement. Agé, la barbe grise, il est assis et ses petits enfants sont agglutinés à ses genoux et s'agrippent à sa robe, le couvant d'un regard possessif: ils quêtent son attention. Les chroniques et livres d'histoires ne sont pas de reste. Dans un manuscrit du XIV ${ }^{e}$ siècle, le père de Philippe Auguste, la couronne sur la tête, prend tendrement son bébé dans les bras à la naissance, tandis que la mère se remet, endormie dans son lit ${ }^{26}$; à l'évidence, cela n'entame en rien sa majestueuse dignité royale. Aux yeux d'un lecteur du XIVe siècle, rien d'étonnant à ce qu'un père, même de la plus haute noblesse, puisse tenir en ses bras un bébé, sans que rien dans le contexte de l'image puisse laisser croire qu'il faut y voir davantage un geste de "reconnaissance" qu'un simple mouvement d'affection et d'émerveillement devant un nouveau-né, futur roi il est vrai. 
C'est sans doute à l'image de tels pères que les artistes du XVe siècle peignent Joseph assis par terre son bébé étroitement serré dans ses bras, tandis que lit la Vierge confortablement installée dans son lit de gésine.

Mais ce sont là, nous dira-t-on, enfances de milieux sociaux élevés. Les vies de saints, parfois issus de milieux sociaux plus modestes, sont pourtant tout autant porteuses d'informations sur les relations affectives positives père-enfants. Saint Eustache se désole, les mains tordues d'angoisse, en voyant ses petits enfants emportés qui par un lion qui par un loup, dans la Légende dorée, que reprennent vitraux et images des livres d'heures: lequel sauver en priorité? Assis au chevet de son épouse qui vient d'accoucher, le père du futur saint Amand, dans un ouvrage enluminé au $\mathrm{XI}^{\mathrm{e}}$ siècle ${ }^{27}$, lève les bras dans son allégresse lors de la naissance de son premier enfant, un garçon (évidemment...). On nous opposera encore qu'il s'agit là de pères modèles, qui ont d'ailleurs formé des enfants modèles: ils ne sont pas devenus saints sans raison... Cependant, d'autres sources encore fournissent des informations positives. Les livres de miracles, davantage que tous autres, mettent en évidence les relations ordinaires entre pères et enfants. On voit ainsi des hommes (les pères?) présenter leur petit enfant devant les autels de sanctuaires dédiés à des saints guérisseurs d'enfants ${ }^{28}$. D'autres, comme dans les Cantigas de Santa Maria, au XIII ${ }^{\mathrm{e}}$ siècle, emmènent leur bébé malade en pèlerinage ${ }^{29}$, accompagné de leur femme, ou offrent à leur jeune fils bien-portant une mule. Lorsque le fils adolescent quitte sa famille pour aller chercher fortune, c'est prioritairement à son père qu'il fait ses adieux, non à sa mère, demeurée en retrait.

14 Enfin, les images isolées du père et ses enfants, qui ne soutiennent aucun texte particulier, sont d'autant plus intéressantes pour l'historien que, la plupart du temps, nulle intention symbolique et nul message ne sont par leur intermédiaire propagés par l'artiste. La famille est un motif décoratif comme un autre au sein d'un paysage, qu'elle anime ; la famille paysanne, si rarement figurée dans les images destinées au plaisir des yeux de la haute noblesse, reprend alors ses droits. Elle apparaît dans un armorial peint en Auvergne au milieu du $\mathrm{XV}^{\mathrm{e}}$ siècle $^{30}$. Un trio, le père, la mère et le petit garçon, partent vraisemblablement au marché du bourg fortifié tout proche. Le père est lourdement courbé sous le faix, la mère chargée de paquets, l'enfant ne porte rien. S'il accompagne ses parents, ce n'est sans doute pas seulement pour ne pas rester seul à la maison. Il revient au père, comme on le voit dans le fabliau du Vilain de Farbus, au XIII siècle, d'emmener son fils à la foire et de lui enseigner les "trucs" de la vente. Dans la majorité des images d'enfants au travail en famille, en effet, le père semble jouer le rôle essentiel d'initiateur aux gestes du métier qu'adoptera ensuite son descendant.

le père initiateur et éducateur

15 Les enfants accompagnent souvent les adultes au travail. Nécessairement, dans ce contexte, le père apparaît amplement. Il semble ainsi enseigner son métier à ses enfants, fils et filles, comme dans un calendrier des ateliers de Simon Bening où les gamins des deux sexes, assis à proximité, regardent leur père griller les soies du cochon qu'on vient de sacrifier ${ }^{31}$. L'initiation du métier n'est pas le seul enseignement que prodiguent les pères de milieux sociaux populaires; ces derniers doivent aussi s'occuper de l'éducation morale de leurs rejetons, par l'exemple ou par leçons. Ainsi emmènent-ils leurs jeunes enfants aux fêtes ${ }^{32}$ et, dans une hotte, leurs bébés aux charivaris ${ }^{33}$ où leurs cris doivent ajouter au désordre. La capacité à éduquer leurs enfants leur est pleinement reconnue : ainsi, Berthold de Ratisbonne exige d'eux qu'ils manient la badine pour empêcher leurs enfants de dire des gros mots ${ }^{34}$. Une historiette 
du XIII siècle présente le motif «dou pere qui chestoie [éduque] son enfant $»^{35}$; l'illustration, en deux parties, montre qu'il s'agit d'un laboureur: la première case figure le père tenant l'enfant par le poignet, la seconde les hommes poussant la charrue.

De fait, et presque toujours en l'absence de la mère, qui joue alors le rôle du tiers exclu, l'enfant, notamment le garçon - du moins dans les images ${ }^{36}$ - accomplit un certain nombre d'activités professionnelles avec son père; dans les enluminures, comme sans doute dans la réalité, l'enfant se retrouve en tête à tête avec son père de sorte qu'à cette occasion leur relation ne peut que s'intensifier ou s'enrichir. Pères et fils vont ensemble aux champs, comme en témoigne une enluminure figurant un accident d'enfant tombé sous la charrue, dans un livre d'Ovide enluminé au XIV ${ }^{e}$ siècle ${ }^{37}$. Ils travaillent ensemble aux moissons dans Le repos de la sainte Famille, un tableau de Patinir, en Flandre, au $\mathrm{XV}^{\mathrm{e}}$ siècle. Tardivement, à la fin $\mathrm{du} \mathrm{XV}^{\mathrm{e}}$ siècle, les enlumineurs imaginent de figurer, dans les calendriers peints des manuscrits, des petits garçons essayant de grimper dans la cuve de vendanges avec l'adulte, sans doute le père, et un gamin plus âgé collaborant avec lui à la tonte des ovins: l'enfant maintient immobile l'animal en lui tenant les pattes arrière ${ }^{38}$. Un autre motif classique des années 1495-1530, dans les manuscrits enluminés comme dans les tapisseries flamandes, est celui du gamin qui ramasse le petit bois et les branches écotées pendant que son père coupe du bois de chauffe, durant les mois d'hiver ${ }^{39}$. On voyait déjà ce motif dans les œuvres morales de Valère Maxime illustrées au XVe siècle. Les hommes abattent un chêne dans l'enclos de l'église, les enfants, à l'évidence des garçons, ramassant les glands et les branchettes écotées ${ }^{40}$. Dans les boutiques des artisans, pères et enfants sont aussi rassemblés, plus souvent en présence de la mère. Le père menuise et l'enfant ramasse les copeaux dans une œuvre de Jean Bourdichon figurant, dans les Quatre états de la société, "le Travail" vide les entrailles des cochons devant son petit garçon qui gonfle une vessie en guise de ballon, image appréciée des artistes ${ }^{42}$. Même les sans-emploi "travaillent" avec leurs enfants: les pères les emmènent tous mendier; c'est un topos de l'iconographie médiévale que de voir une noble femme donner l'aumône au pauvre père chargé d'enfants, un sur les épaules, un nourrisson dans un porte-bébé de poitrine, un dernier accroché à sa robe, comme dans les Heures de Jeanne de Navarre... ${ }^{43}$

Dans les milieux aristocratiques, qui ne sont pas soumis à l'obligation de travailler de leurs mains, le père joue également le rôle d'éducateur. Mais il n'enseigne évidemment pas les mêmes choses. Dans les livres moraux de Valère-Maxime copiés au $\mathrm{XV}^{\mathrm{e}}$ siècle, en présence ou non de la mère, il initie ses enfants à l'idée de pauvreté... en leur montrant les trésors d'orfèvrerie qu'il a précautionneusement enfermés dans le coffre ferré du château ${ }^{44}$, à moins que, pour lui enseigner les réalités sociales, il ne présente à son fils, dans la cour du domaine, un vrai paysan, au titre « de la mutation des muers et de fortune $\aleph^{45}$. De même, il enseigne à son fils et à sa fille, deux bambins qui s'accrochent à sa robe et à son aumônière, les êtres et les objets qui fondent le domaine seigneurial : un paysan soumis, assis par terre à ses pieds, et des outils agricoles ${ }^{46} \ldots$ Bref il leur fait découvrir le monde qui les entoure. Il s'inquiète aussi des bonnes mœurs de ses enfants, réprime leurs mauvais penchants; on en trouve un joli exemple dans la vie de saint Louis lorsque le futur saint, à l'âge de neuf ans, vola un chapon dans la cuisine du roi son père - pour le donner à un pauvre; pris sur le fait, il fut conduit manu militari par le cuisinier devant son père pour y être interrogé. Mais lorsqu'on souleva la robe de l'enfant, le chapon dissimulé s'était transformé en roses et le roi «commanda au 
maître-queux que dorénavant il le laissât prendre ce qu'il voulait pour les pauvres, connaissant la bonne intention et ferveur de son fils». À la fin du $\mathrm{XV}^{\mathrm{e}}$ siècle, l'enlumineur qui illustra l'épisode dans un manuscrit destiné à une duchesse de Bourbon, choisit de représenter la scène du vol et du don du chapon mais non la comparution devant le père justicier ${ }^{47} .$. De fait, la punition paternelle est une scène rarissime dans l'iconographie.

Dans les milieux aisés, lettrés et aristocratiques, le père, lorsqu'il le peut, ne se contente pas non plus de confier l'éducation intellectuelle de ses enfants à un pédagogue ou à sa femme : on le voit lui aussi apprendre seul à lire à sa fille, la férule à la main - un geste symbolique et non pas réaliste ${ }^{48}$ - comme Philippe, père de sainte Eugénie, dans un Miroir historial de Vincent de Beauvais ${ }^{49}$. C'est le père qui, dans les «Dyalogues dou pere et dou filz », dispense avec amour son enseignement à l'enfant attentif, voire dubitatif ${ }^{50}$ malgré les belles formules d'affection que le père lui prodigue dans le texte : «Beau Fils » ou «Treschier et tresamé filz, pour la grant affection et amour paternel que a toy ay... $\wedge^{51}$. Tel le chevalier de la Tour Landry enfin - pédagogue bien connu-assis en chaire, et en présence de son épouse, il convoque ses filles "petites et de sens desgarnies", pour leur enseigner les règles de bonne vie chrétienne ${ }^{52}$. Dans les milieux princiers ou royaux, les pères gardent leurs fils à leur côté lorsqu'ils reçoivent des dignitaires. Mais l'éducation physique et le sport relèvent aussi, plus naturellement encore, de sa compétence ; ces activités sont essentielles pour espérer garantir la survie ultérieure des jeunes au combat. Ainsi emmène-t-il ses enfants à la chasse, l'enfant à califourchon derrière lui, ou juché sur un poney à sa taille ${ }^{53}$, partageant ainsi avec eux les plaisirs de la promenade à cheval. Et c'est devant l'assemblée des hommes du château, et sans doute en présence du père, sans qu'une seule femme soit présente, que les adolescents s'exercent au lancer du poids et du javelot ${ }^{54}$ ou au tir à l'arc ${ }^{55}$ : le sport, déjà, domaine réservé du père?

le père soucieux et salvateur

19 Les trois derniers siècles du Moyen Âge ne sont pas avares en représentations d'inquiétude paternelle, qui trouve à s'exprimer dans les difficultés nombreuses de la vie quotidienne : maladie, mort subite ou accidentelle des enfants hélas ne manquaient pas et sont d'ailleurs souvent figurées tant dans les recueils illustrés de miracles que dans les ex-votos. L'attitude des pères y est fort instructive. Lors des maladies graves de leurs rejetons, ils prennent soin d'eux et les accompagnent en pèlerinage. Du couple parental, c'est le père qui est mis à l'honneur, au premier plan de l'image, au XV' siècle, dans les miracles de saint Louis, dans le processus de guérison d'un petit Gautier souffrant «d'une enfleure au col grosse comme un œuf de geline », c'est l'homme seul qui accompagne en pèlerinage de santé un jeune garçon aveugle ${ }^{56}$. Dans le risque, le père joue un rôle essentiel : il prie à genoux, avec ferveur, les bras dressés au ciel, de l'autre côté de la porte derrière laquelle son épouse donne le jour à un enfant ${ }^{57}$.

Dans la mort, le rôle du père est plus fortement encore souligné par les artistes et l'on se demandera dans quelle mesure ces derniers se font là simples observateurs de la réalité ou s'ils l' "arrangent" conformément à un idéal médiéval de comportement paternel. Quoi qu'il en soit, l'observation des images délivre une information de grand intérêt. Ainsi, dans un ex-voto italien peint par Matteo di Giovanni (actif 1452-†1495), si c'est la mère qui se précipite pour sauver l'enfant noyé dans le bief d'un moulin, c'est le père qui supplie le ciel, mains jointes ${ }^{58}$. De même dans un autre ex-voto peint par Sano di Pietro (1405-1481) qui figure un enfant de 18 mois noyé dans une cuve à Aquila: 
tandis que la mère sort le bambin de l'eau, c'est le père qui prie mains jointes, accompagné d'une autre femme, voisine ou nourrice ${ }^{59}$. Penché sur son garçonnet miraculé après avoir été attaqué par un chien, c'est encore le père qui prie, vu par Simone Martini ${ }^{60}$. Courbé au-dessus de la surface d'une rivière dans laquelle vient de disparaître son petit garçon, dans les Cantigas de santa Maria, au XIII ${ }^{\mathrm{e}}$ siècle, le père joint ses mains en prière tandis que la mère se griffe les joues en signe de deuil ${ }^{61} \ldots$ Dans un autre miracle de san Agostino peint par Simone Martini, tandis que la mère s'arrache les cheveux, ce sont encore des hommes (dont le père ?) qui ramassent l'enfant tombé d'un balcon et qui prient pour lui. Les peintures et enluminures figurant des miracles d'enfants attribuent systématiquement au père ce rôle d'intercesseur. Encore dans la prédelle d'un autel dédié à saint Louis de Toulouse, on voit qu'un père (?) brandit vers le ciel et le saint une statuette à son image ${ }^{62}$, qu'il lui voue, en échange de la vie de son fils étendu sur son lit de mort et entouré de deux femmes dont l'une s'arrache les cheveux en signe de deuil et, tête renversée en arrière, hurle sans doute elle aussi des supplications au ciel ${ }^{63}$.

21 La même attitude est adoptée par le père au sanctuaire dans lequel on présente la dépouille de l'enfant. Toujours dans les Cantigas, devant l'autel de la Vierge où l'enfant mort est porté par ses parents espérant un répit, c'est la mère qui brandit l'enfant vers la sainte statue tandis que prie le père, parfois accompagné des hommes de l'assistance ou de la famille ${ }^{64}$. Ainsi, la prière du père semble jugée essentielle à l'obtention du miracle. Huit ou dix images ne peuvent en aucun cas permettre de fonder une théorie, mais l'identité des postures paternelles est frappante: dans le danger, devant la mort imminente, outre l'emploi de sa force physique qui lui permet de sortir de la rivière le petit noyé et de porter l'enfant accidenté dans les bras ${ }^{65}$, il est investi d'une responsabilité spirituelle et a un rôle personnel à jouer, celui de la prière fervente et ostentatoire qui appelle au miracle ${ }^{66}$, tandis que la mère tantôt s'active plus concrètement à sauver son enfant tantôt parait, au contraire, paralysée par l'angoisse. Et qui sait si l'appel au miracle n'était pas jugé plus important encore que le geste du secouriste ?67

le père affectueux

Les gestes d'affection paternels sont une constante de la représentation paternelle dans les manuscrits médiévaux et le modèle du père aimant est bien antérieur au bas Moyen Âge: on voit ainsi, dans un codex fameux $\mathrm{du} \mathrm{VI}^{\mathrm{e}}$ siècle, la Genèse de Vienne, Joseph se plier en deux pour embrasser son bébé avant de le quitter puis se retourner dans son chagrin de se séparer de lui ${ }^{68}$. Dans les moments paisibles, le père ne manque pas de prodiguer à son enfant caresses et gestes d'affection: les artistes d'alors s'attachent également à les représenter, soucieux sans doute de stimuler ainsi l'émotion ou l'attendrissement de leurs lecteurs qui apprécient de plus en plus, au fil du Moyen Âge, les scènes de tendresse familiale ${ }^{69}$. Ces dernières sont parfois délicates à interpréter : les gestes changent de sens. Le contact physique entre père et enfant prend à l'occasion un caractère inattendu: on voit ainsi saint Louis, image du père modèle qui s'occupe chaque soir de ses enfants en leur faisant la lecture et en leur faisant réciter leurs heures, tenir par la gorge l'un de ses fils qui prient à genoux devant lui lors d'une fête religieuse ${ }^{70}$. C'est sans doute là une caresse et non une menace... Plus explicites, parce que la signification du geste n'a pas varié, on voit, dans les Cantigas de santa Maria, un manuscrit espagnol du XIII ${ }^{e}$ siècle, le père qui offre à son jeune fils une mule lui entourer l'épaule de son bras. Dans un calendrier padouan du XIV ${ }^{e}$ siècle figurant le 
signe du verseau, pendant que la mère s'échine à transporter le bois de chauffage vers le foyer, c'est le père qui, tranquillement assis au coin du feu, caresse la joue de son petit garçon tout en bavardant avec lui, si l'on en juge au mouvement symptomatique qu'il fait de l'autre main ${ }^{71}$; l'enfant lui-même lui pose la main sur le bras qui caresse : bel échange de tendresse. Une main posée sur l'épaule de son fils est le geste le plus couramment figuré par les enlumineurs ${ }^{72}$. Cette gestuelle est aussi celle de la mère.

Ce prototype du père oisif mais s'occupant de son enfant au coin du feu perdure dans l'iconographie flamande des $\mathrm{XV}^{\mathrm{e}}$ et $\mathrm{XVI}^{\mathrm{e}}$ siècles. On voit ainsi souvent, tandis qu'œuvre la mère au bien-être du foyer, le père se chauffer dans son fauteuil, l'enfant assis sur le bord de la sole de la cheminée, à ses côtés ${ }^{73}$. À la veillée, il y a toujours un enfant près du père, levant les yeux vers lui, comme dans les illustrations des traités médicaux ${ }^{74}$. L'intimité corporelle entre père et enfant est de plus en plus accentuée au fil du Moyen Âge. Dans les Cantigas, au XIII ${ }^{\mathrm{e}}$ siècle, les pères prennent leurs bébés dans les bras $^{75}$. On a vu, dans un manuscrit du XIV siècle, le père de Philippe Auguste prendre son bébé dans ses bras. Joseph fait de même dans un livre d'heures du XVe siècle : assis par terre, il serre étroitement son bébé dans ses $\operatorname{bras}^{76} \ldots$ Dans une étonnante gravure de la seconde moitié du $\mathrm{XV}^{\mathrm{e}}$ siècle, Joseph, tout sourire, se met à quatre pattes et se cache à moitié derrière la banquette d'un jardin comme pour faire "coucou" à son Enfant avant de lui tendre une pomme, qu'il ramasse sur l'herbe ${ }^{77}$. Notons que dans l'esprit de ces artistes, Joseph n'a (encore) rien d'un saint mais tout d'un homme ordinaire: contrairement à Marie, il n'a pas droit à l'auréole. Nous le considèrerons dans ce cas comme un bon modèle de père, et non comme un père bon parce que saint. Autre et nouveau modèle $\mathrm{du}$ bon père, au $\mathrm{XV}^{\mathrm{e}}$ siècle, celui de l'homme sauvage; longtemps présenté comme effrayant, il devient subitement, comme s'il n'était plus possible d'envisager la paternité autrement que positivement, l'image même du bon père qui, tel Joseph, joue avec son bébé et le laisse lui tirer les poils tout en le tenant dans ses bras.

le père aimé

Cette affection que manifeste le père vis-à-vis de ses jeunes enfants, ces derniers le lui rendent bien, qui lui prodiguent également des gestes d'affection. Le $\mathrm{XV}^{\mathrm{e}}$ siècle, tout à fait dégagé des contraintes formelles qui auparavant standardisaient et stéréotypaient les images, fourmille de scènes de tendresse et d'amour filiaux. Au premier rang, l'Enfant Jésus, bon fils, s'amuse tout bébé à tirer la barbe de son père, comme on le voit dans un bas relief en bois sculpté doré et polychrome allemand, daté de 1500, exposé au Musée national du Moyen Âge ${ }^{78}$. Dans l'ouvrage d'Aristote destiné à la famille du Fou, les enfants paraissent regarder leur père avec autant d'affection que de sentiment possessif au chapitre «de l'amour... du père au fils ${ }^{79}$. Même regard possessif et empreint d'une confiance inquiète dans le portrait de Francesco Sassetti et de son fils Theodoro (?), peint vers 1485-89 par Domenico Ghirlandaio ${ }^{80}$; l'enfant s'accoude à la cuisse de son père et lève son profil pur vers lui. Plus d'affection encore entre l'enfant de la même famille, tant les deux se ressemblent? - et son grand-père dans le très célèbre «Portrait d'un vieillard et de son petit-fils » peint par le même artiste dix ans plus tard et conservé au Musée du Louvre. Ce regard de l'enfant vers le père devient un thème si répandu qu'on le trouve dans tous les médias du temps, enluminure, peinture, gravure $^{81}$. Il n'est pas seulement stéréotypé : il est à l'évidence considéré alors comme une caractéristique de la relation fils-père. Dehors, les pères tiennent leur enfant par la main, qu'il s'agisse de pères bibliques, dans une image stéréotypée que l'on retrouve 
dans toutes les bibles illustrées du XIII ${ }^{e}$ siècle ${ }^{82}$, ou de pères vraisemblables, acteurs de romans médiévaux ${ }^{83}$. Déjà, à l'aube du XIII ${ }^{\mathrm{e}}$ siècle, comme dans le Psautier de la reine Ingeburge, lors de la fuite en Égypte, c'est Joseph qui marche en portant son enfant dans les bras tandis que Marie se repose, assise sur son âne ${ }^{84}$.

les pères cruels

On cherchera en vain, dans l'iconographie médiévale, les images de violences paternelles dans un contexte vraisemblable. Les seuls pères cruels sont les figurations, tardives (fin du XV $\mathrm{XV}^{\mathrm{e}}$-début du XVI ${ }^{\mathrm{e}}$ siècle), de Saturne dévorant ses enfants ou des ogres et croquemitaines qui sont, presque toujours, de sexe masculin. Les méchants pères existent, mais ils sont rarement figurés, moins que ne l'est la mère cruelle. Le mauvais père n'est d'ailleurs pas nécessairement celui qui bat son fils, mais celui qui lui donne le mauvais exemple: dans la gravure illustrant le Stultifera navis de Sébastien Brant, publié à Bâle en 1497 et immédiatement diffusé à travers toute l'Europe, le chapitre «des mauvais exemples donnés par les parents » figure le père qui tient un tablier de trictrac (son premier vice, le jeu), et menace l'épouse d'un pot de vin (second vice, la boisson) fêlé (dernier défaut, la violence) ; le gamin, un biberon au poing, regarde sa mère qui n'en peut mais.

Une des très rares images de violence paternelle est issue d'une version illustrée au $\mathrm{XV}^{\mathrm{e}}$ siècle d'un Livre des propriétés des choses, de Barthélemy l'Anglais, et il serait délicat d'interpréter au pied de la lettre, si l'on puit dire en parlant d'une image, cette enluminure figurant le père sur le point de frapper d'une verge son fils ${ }^{85}$, tandis que la mère, toujours protectrice, pose une main compatissante sur son épaule, tout en détournant la tête cependant car elle n'a pas le droit de s'opposer à la décision paternelle. Sans doute faut-il admettre comme une évidence le fait que c'est au père, et non à la mère, qu'est ici imputé le rôle traditionnel de "père Fouettard". Mais l'image ne signifie pas pour autant que les pères battaient les enfants à tour de bras et à tout moment ni même qu'on leur conseillait de le faire: elle illustre une sentence de l'enclyclopédiste, qui dit simplement qu'il est inutile de frapper les enfants pour les faire obéir avant qu'ils n'aient atteint l'âge de raison, sept ans ${ }^{86} \ldots$ Certes, il est sousentendu qu'on peut le faire ensuite mais là où le texte autorise la nuance, l'image, simplifiée pour être immédiatement comprise, le trahit presque nécessairement.

L'image, qui peut trahir le texte, travestit aussi volontairement les réalités sociales ; on en trouvera un exemple dans la traduction, vue par des artistes chrétiens, de la paternité juive. Quand le père est présenté comme cruel, c'est qu'il s'agit d'un juif : ainsi, on voit assez souvent enluminée l'histoire du père de famille juif qui découvre que son fils est allé communier avec des petits chrétiens et qui sans états d'âme le jette au feu dans un four de verrier en punition, alors que la mère se désole ${ }^{87}$. Mais ce sont là des images-repoussoir, destinées à stigmatiser les juifs en leur attribuant des attitudes justement rejetées par les "bons chrétiens"... Inutile de dire que lorsque les juifs euxmêmes se font représenter en pères de familles, ils n'ont rien en commun avec la caricature qu'en donnent les artistes chrétiens et que, comme tout un chacun, ils se comportent en bons pères envers leurs enfants, leur posant la main sur la tête en une caresse protectrice classique, comme dans la Haggada de Sarajevo ${ }^{88}$, au XIII ${ }^{\mathrm{e}}$ siècle. Les relations père-fils sont même fortement valorisées dans la vie quotidienne, qui les ritualise, notamment à l'occasion de la fête de Pâques ou, de façon plus intime encore, lors de la première journée d'école des enfants lors de laquelle il incombe au père d'emmener son fils à l'école dans ses bras et enveloppé dans le manteau paternel, au 
point qu'on a pu - très exagérément sans doute - comparer le père juif à la Vierge Marie à l'Enfant $!^{89}$ Des images témoignent de ce beau geste ${ }^{90}$.

pères et filles

28 Et les filles? Dans les familles juives, pères et filles avaient sans doute les mêmes relations affectueuses, si l'on en juge à la superbe lamentation de Liézer de Worms pour sa femme et ses deux filles assassinées, en 1196, où ce père parle avec désespoir de sa fille aînée de 13 ans, Belet, qui non contente d'être pieuse et savante, «faisait son lit et lui enlevait ses chaussures chaque soir (...), tissait, cousait, brodait (...) et s'asseyait à ses pieds pour l'écouter dire la Tora», et de sa benjamine de six ans, qui non seulement maitrisait les travaux d'aiguille mais qui «l'amusait et chantait $»^{91}$... Cependant, les filles sont absentes de l'inventaire des images de pères et leurs enfants, tant chez les juifs que chez les chrétiens, ce qui ne signifie nullement que les pères ne s'en occupaient pas: les lettres de rémission corrigent amplement sur ce point l'image, faillible, stéréotypée, qui tout au long du Moyen Âge privilégie le rapport père-fils, ou mère-fils, à celui qu'entretenaient tout autant les parents à leurs filles. Les filles ne peuvent guère apparaître, hormis dans les arbres de consanguinité où il est impossible d'élaguer les branches féminines... Dans les encyclopédies, l'enfant du motif des «âges de la vie de l'homme» (et non de la femme...) est par définition de sexe masculin. Parmi les multiples tout-petits que portent les pères, nul ne peut dire si quelques fillettes se glissent dans le lot: les bébés, comme on le voit dans le motif des arbres de consanguinité, ont un vêtement unisexe. Il faut donc une image illustrant un texte circonstancié pour qu'avant l'âge de la robe féminine et des cheveux longs, l'on reconnaisse une fille avec son père. C'est le cas dans les livres de miracles - mais si père et enfants sont alors mis en scène c'est souvent à la suite d'un défaut de surveillance parental et le rapport se place alors presque nécessairement sur un plan négatif: Marotte, qui se noie pendant que son père, sorti, a confié sa garde au frère aîné, qui la laisse toute seule au bord de l'eau ${ }^{92}$.

Plus généralement, dans l'iconographie, les filles ne sont presque jamais associées à leur père car l'image, toujours stéréotypée, tend à associer séparément père et fils d'une part, mères et filles de l'autre. On peut voir plusieurs explications à ce fait. La première est l'obéissance aux théories médicales ou encyclopédiques. Le père aime celui de ses enfants qui lui ressemble le plus, dit au XIII ${ }^{\mathrm{e}}$ siècle Barthélemy l'Anglais : on n'imagine pas - dans la représentation figurée du moins, qui pour être immédiatement décodable se doit d'être toujours simplifiée - que ce puisse être une fille. Telle mère, telle fille, dit un proverbe (évidemment médiéval) : voilà aussi pourquoi les peintres et les enlumineurs tendent systématiquement à représenter les filles du côté de la mère et les fils de celui de leur père. Les artistes, suivis en cela par leurs commanditaires, les pères et mères réels, séparent donc quasi systématiquement les sexes dans la représentation des familles réelles ou imaginaires: ainsi, dans un livre d'heures à proverbes daté du milieu du $\mathrm{XV}^{\mathrm{e}}$ siècle, on voit qu'autour d'une table familiale les garçons sont rangés en rang d'oignon et par ordre de taille décroissant du côté du père, et les filles de la même façon du côté de la mère ${ }^{93}$; sans doute s'agit-il de la famille de Job, dont les mésaventures sont amplement développées dans le volume. D'innombrables images répondent à ce modèle, qui convient pour l'enluminure française et italienne, chrétienne ou juive : ainsi, dans la représentation de la famille de Job, dans un manuscrit juif italien du XIV e siècle, Job est assis en chaire au centre de l'image, les hommes de sa famille à sa droite, les femmes à gauche ${ }^{94}$ - le côté féminin... 
Ce prototype vaut aussi pour le motif des "arbres de consanguinité" : mère et père se tiennent au pied de l'arbre, dans une Somme rurale de Jean Boutillier, ouvrage de droit coutumier, réalisée à Bruges en $1471^{95}$. Du côté de la mère, les branches féminines, couvertes de petites filles, du bébé aux adolescentes et aux jeunes adultes. Du côté du père, les garçons ${ }^{96}$.

Dans la représentation des familles royales, fréquentes dès le XIV ${ }^{e}$ siècle dans les manuscrits qui leur étaient destinés, le roi et la reine trônent au centre de l'image, les petits garçons debout du côté de leur père, la ou les petites filles du côté de leur mère : c'est ainsi qu'est figurée la famille de Philippe le Bel, dans un recueil de fables persannes, le Livre de Dina et Kalila ${ }^{97}$, ou de Charles V dans des Éthiques d'Aristote ${ }^{98}$ ou un Rational de Guillaume Durand ${ }^{99}$. S'il n'y a que des garçons, ils se tiennent seulement du côté de leur père. Dans le portrait de famille, genre pictural qui se généralise à la fin du $\mathrm{XV}^{\mathrm{e}}$ et au XVI $\mathrm{XI}^{\mathrm{e}}$ siècle, et qui figure le plus souvent la famille en prière, les filles prient derrière leur mère, et les garçons derrière leur père. Si la famille n'a qu'un enfant unique, et si c'est un garçon, ce dernier est agenouillé derrière son père, non sa mère ${ }^{100}$. Comment interpréter ce type de représentation? En la prenant au pied de la lettre, comme si les mères ne s'occupaient que des filles et les pères que des garçons? Cette solution ne résiste pas à l'épreuve des autres sources. Il faut plutôt y voir l'expression jusque dans les images d'un besoin de classification typiquement médiéval, qui fait absolument fi des réalités quotidiennes.

Au demeurant, d'autres modèles de représentation existent, du moins lorsqu'un enfant unique seulement est mis en scène avec ses parents. Il est alors assis du côté de la mère, surtout si l'on a voulu représenter le père en tant que roi avant de l'être en tant que père, comme dans le Cartulario de Tojos Outos (Coruña), aux XII ${ }^{\mathrm{e}}$-XIII ${ }^{\mathrm{e}}$ siècles ${ }^{101}$. Mais prenons garde au contexte, qui peut expliciter la position de l'enfant entre les deux parents. Dans les images familiales des manuscrits juifs, ouverts à la page de la fête de Pâques, on voit toujours un garçon assis entre le père et la mère : mais c'est pour répondre ici au rituel festif. Dans un psautier-livre d'heures flamand du XIII ${ }^{\mathrm{e}}$ siècle, destiné à Yolande de Soissons, le (vrai) père de famille, possesseur du manuscrit, figure au début du volume avec le reste de sa famille, son épouse et ses deux enfants, fils et fille ${ }^{102}$. La représentation, symbolique, adopte une autre mise en scène, non moins révélatrice: il pose la main sur l'épaule de sa femme qui enlace ses deux enfants, une main sur l'épaule de chacun. Par ce geste, il délègue ses pouvoirs à l'épouse dans l'éducation des enfants. Ce qui ne signifie sans doute nullement qu'il se décharge de cette tâche à son profit! Dans les familles plus ordinaires, où l'enlumineur ne cherche pas à classifier mais à donner un semblant de réalisme à une illustration de roman, les enfants des deux sexes se tiennent, comme par exemple à table, du côté de la mère et non du père ${ }^{103}$.

Père et mère, des rôles séparés?

Faut-il donc penser que l'image n'est pas un bon indicateur des relations pères et enfants? Que non pas. Mais il ne faut pas sous-estimer les capacités des hommes médiévaux à l'abstraction et à la catégorisation et nous devons, nous aussi, reclasser les images et les analyser selon la catégorie à laquelle elles appartiennent. L'intéressant est le nombre important de représentations de tendresse paternelle, et pas uniquement joséphiques, qui peuplent les pages des manuscrits médiévaux familiaux. Ne regardons plus les images avec notre regard d'hommes $d u X X^{e}$ siècle, qui n'ont guère de temps, contrairement au Moyen Âge, à accorder à leurs enfants, mais avec celui des temps 
médiévaux, où le temps chômé abondait et n'était pas distrait par la télévision ou la civilisation des loisirs, et où l'on n'avait pas sans doute pas encore inculqué aux garçons la séparation des tâches en matière d'élevage des enfants, où l'homme avait le droit de se laisser aller à l'expression de son sentiment "maternel" envers les enfants et même le devoir de s'occuper d'eux, y compris matériellement, quand son épouse était absente ou morte, ou encore chaque fois qu'elle était malade ou dans l'attente des relevailles, ce qui lui arrivait au moins un an sur deux... Et s'il faut, pour conclure, reconnaître un rôle particulier au père, c'est celui du jeu : presque jamais, sans doute parce qu'elle est bien trop occupée aux soins du ménage, du filage, ou des derniers-nés pour cela, on ne voit la mère jouer avec ses enfants ${ }^{104}$ : c'est là le rôle du père qui tend à son enfant une toupie, tel Joseph, ou lui gonfle une vessie de porc en guise de ballon et brandit de jolies chaussures qu'il leur offre, les gamins tendant les bras en l'air pour s'en saisir, comme dans la tapisserie-calendrier du château des Sforza, à Milan, datée de 1504-1508 ${ }^{105}$.

Si, dans les images, c'est Ève qui balance le berceau du bout du pied, et non le père, c'est qu'Adam a les siens fermement ancrés dans la boue qu'il bêche pour sa punition tandis qu'Ève, selon le proverbe médiéval, file tranquilement, assise, surveillant son bébé. Rien ne dit qu'à la veillée les pères n'aimaient pas balancer leur dernier-né : ils leur composent et leur chantent bien des berceuses, pourquoi ne berceraient-ils pas leur enfant en même temps? Si, dans les images, les pères ont des rôles distincts de ceux des mères, ce n'est guère que dans le détail et pour des raisons surtout techniques de surcroît. Ainsi, pères et mères accompagnent leurs enfants en pèlerinage de santé, portent, lorsqu'ils sont en voyage, leur bébé au berceau en équilibre sur la tête ou dans le dos, voire leurs jeunes enfants dans les bras, ou sur les épaules. Mais les pères en portent souvent davantage que les mères: question de force musculaire; et ils se chargent parfois de porter le berceau, tandis que la mère garde le nourrisson dans ses bras $^{106}$. On voit, au XIII siècle, calés dans une hotte d'osier en guise de porte-bébés, deux enfants dans le dos d'un père de famille fuyant l'Apocalypse ${ }^{107}$ ou, au XIV ${ }^{\mathrm{e}}$ siècle, un autre père en tenant un bébé en robe au bras tandis que de l'autre main il tient le poignet d'un garçonnet; la mère guide un âne bâté porteur de deux petits enfants sans doute trop jeunes pour marcher.

Certes, il y aura toujours plus d'images de tendresse ou d'attention maternelles à l'enfant que d'images paternelles, mais c'est aussi qu'un nombre incalculable d'illustrations se focalisent sur des gestes que les pères ne sauraient accomplir. Les hommes n'accouchent pas... Sauf dans un cas! La "couvade" est représentée dans un Livre des Merveilles de l'extrême fin du $\mathrm{XV}^{\mathrm{e}}$ siècle et les nobles lecteurs des manuscrits pouvaient y voir leur semblable au lit, le bébé dans les bras, ragardant une servante lui apporter le traditionnel bouillon de poule de l'accouchée ${ }^{108}$. De même, l'allaitement au sein leur est à jamais interdit... ce qui ne les empêche pas de donner le biberon, ainsi qu'on le voit dans une bible historiée du XIV siècle $^{109}$. Ce qui importe, c'est que pères et mères sans distinction prodiguent aux enfants soins et gestes d'affection, et même regards d'amour envers leur nouveau-né, tel celui que pose, indiscutablement, l'époux de Griseldis dans une fresque anonyme peinte au château de Roccabianca, à Milan, à la fin du XV siècle. Nous pensions, en 1985, que la seule tâche que les pères médiévaux auraient refusé d'assumer est le lavage des changes «breneux » de leurs enfants. Nous n'oserions même plus l'écrire aujourd'hui, inquiète à l'idée d'être un jour démentie par l'incontournable réalisme de quelque lettre de rémission dont un père de famille serait, comme souvent, le héros... 


\section{NOTES}

1. Histoire de la famille, sous la dir. d'A. Burguière, Ch. Klapisch-Zuber et al., tome 2, Paris, Hachette, Livre de Poche références, pp. 200-205.

2. Histoire des femmes, sous la dir. de G. Duby et M. Perrot, tome 2, Le Moyen Âge, sous la dir. de Ch. Klapisch-Zuber, Paris, Plon, 1991, pp. 132-135.

3. Où sont passés les pères?, Paris, Fondation pour l'enfance, avril 1995. L'Histoire des pères et de la paternité signale que des enquêtes ont récemment montré qu'en réalité «en dix ans la participation des pères aux soins des enfants et au ménage n'est que de quelques minutes supplémentaires par jour »..., p. 399.

4. M. Daumas, Le Syndrome des Grieux. La relation père-fils au XVIII ${ }^{e}$ siècle, Paris, Seuil, 1990.

5. Id., Ibid., p. 37, exemple connu : «Les Lumières, dit-on, ont inventé le mariage d'amour...»; on appréciera cependant le caractère nuancé ici de cette affirmation.

6. Id., Ibid., p. 19.

7. Sous la plume de S. Melchior-Bonnet, « De Gerson à Montaigne, le Pouvoir et l'amour", voir la p. 56.

8. Nous avons aussi souhaité présenter, dans le livre qui accompagnait l'exposition, le père avant la mère, non pour déprécier le rôle de cette dernière, mais pour remettre à l'honneur celui du père dans la puériculture : $\mathrm{P}$. Riché et $\mathrm{D}$. Alexandre-Bidon, $L$ 'Enfant au Moyen Âge, Paris, Seuil/BNF, 1994, p. 92.

9. Voir les travaux de D. Lett dans D. Alexandre-Bidon et D. Lett, Les Enfants au Moyen Âge, Paris, Hachette, coll. La vie quotidienne, 1997, p. 112.

10. Voir les travaux récents sur les lettres de rémissions, C. Gauvard, "De Grace especial". Crime, État et société en France, à la fin du Moyen Âge, Paris, Publ. de la Sorbonne, 1991.

11. R. Virgoe, Les Paston, une famille anglaise au XVe siècle, Paris, Hachette, 1990, p. 45.

12. Abbé Garnier, «La Nativité vue par les imagiers », Enluminure carolingienne et romane, $\mathrm{n}^{\circ}$ 14, janvier-février 1976, pp. 17-25.

13. Sous la dir. de J. Delumeau et de D. Roche, Paris, Larousse, 1990, pp. 212-213.

14. D. Alexandre-Bidon et M. Closson, L'Enfant à l'ombre des cathédrales, Lyon, PUL-CNRS, 1985.

15. Id., ibid., pp. 208-211.

16. Paris, BNF, ms fr 244 fol. 24. Paris, vers 1480-1485, reproduit dans P. Riché et

D. Alexandre-Bidon, op. cit., p. 196.

17. Paris, BNF, ms latin 1156. Horae ad usum romanum.

18. Publiée fautivement pp. 166-167, republiée sans faute, accompagnée d'une autre berceuse écrite par un père de famille italien, dans $\mathrm{P}$. Riché et $\mathrm{D}$. Alexandre-Bidon, L'Enfant au Moyen Âge, Paris, Seuil/BNF, 1994, pp. 56 et 58.

19. D. Alexandre-Bidon et M. Closson, op. cit., p. 90. Nous l'attribuions naïvement au département des Estampes de la BNF. J'ai eu l'occasion de découvrir depuis, en préparant l'exposition sur L'Enfance au Moyen Âge pour cette prestigieuse institution, que le volume, bien qu'imprimé, était en réalité conservé au département des manuscrits, sous la cote Rothschild II. 3.24 (1841).

20. Voir l'Histoire des Pères, pp. 208-209 et surtout l'article de F. Boepsflug dans Le Pressoir mystique, sous la dir. de D. Alexandre-Bidon, Paris, Éd. du Cerf, 1990. 
21. Par exemple Paris, BNF, ms fr 9140 fol. $102^{v}$, reproduit dans P. Riché et D.

Alexandre-Bidon, op. cit., p. 91, et fr 22534 fol. $80^{\mathrm{v}}$.

22. Valencia, Bibl. Univ., ms 1372 fol. $150^{\mathrm{r}}$.

23. Par exemple Paris, BNF, ms fr 9141 ou fr 22531 fol. $99^{\mathrm{v}}$.

24. Sur cette fonction particulière des images, voir J.-C. Schmitt, «Les images classificatrices », Bibliothèque de l'École des Chartes, tome 147, Paris-Genève, Droz, 1989, pp. 311-341.

25. Paris, BNF, ms fr 22500.

26. Lyon, BM, ms 880 fol. $239^{r}$.

27. Valenciennes, BM, ms 502 fol. $5^{\mathrm{v}}$, reproduit dans P. Riché et D. Alexandre-Bidon, op. cit., p. 95.

28. Paris, BNF, ms fr 5716, Miracles de Notre Dame, France, $X_{I V}{ }^{e}$ siècle.

29. Madrid, Bibl. de l'Escorial, voir le fol. $225^{\mathrm{r}}$.

30. Paris, BNF, ms fr 22297 fol. $344^{\mathrm{r}}$ (château du Broc). Armorial de Revel, Auvergne, milieu du $\mathrm{XV}^{\mathrm{e}}$ siècle.

31. Bruxelles, Bibl. Roy. Albert $\mathrm{I}^{\mathrm{er}}$, Heures de Hennessy, ms II 158, mois de décembre.

32. Par exemple, Paris, Nal 3145 fol. $53^{\mathrm{r}}$, Heures de Jeanne de Navarre, Paris, v. 1336-1340.

33. Paris, BNF, ms fr 146, Roman de Fauvel, France, $X^{\mathrm{e}}$ siècle.

34. Péchés et vertus. Berthold de Ratisbonne, éd. par C. Lecouteux, Paris, 1991, p. 115.

35. Lyon, BM, ms PA 57 fol. $68^{\mathrm{v}}$.

36. Cela ne se vérifie pas aussi systématiquement dans les sources écrites : ainsi, selon ses parrains et autres habitants de son village, Jeanne d'Arc, petite fille, accompagnait son père à la charrue. R. Pernoud, Jeanne d'Arc, par elle-même et par ses témoins, Paris, 1962.

37. Lyon, BM, ms 742 fol. $205^{\mathrm{v}}$.

38. Paris, BNF, ms lat 16827 fol. $19^{v}$ et $15^{v}$.

39. Par exemple Munich, Bayer. Staat. Bibl., Calendrier flamand de Simon Bening, mois de janvier.

40. Paris, BNF, ms fr 6275 fol. $1^{\mathrm{v}}$.

41. Paris, École des Beaux-Arts et Paris, BNF, ms fr 2374, dessin préparatoire.

42. Par exemple, Modène, Bibl. Estense, ms lat 424 fol. $6^{\mathrm{V}}$ ou Paris, BNF, ms fr 1872 fol. $13^{\mathrm{r}}$, reproduit dans P. Riché et D. Alexandre-Bidon, op. cit., p. 74.

43. Paris, BNF, ms Nal 3145.

44. Paris, BNF, ms fr 6185 fol. $140^{v}$.

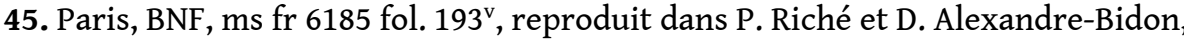
op. cit., p. 96.

46. Paris, BNF, ms fr 22500 fol. $6^{\mathrm{r}}$.

47. Voir le fac similé partiel du manuscrit : Saint Louis, roi de France, Paris, Chêne, 1990, où texte et image de l'épisode sont reproduits.

48. Voir F. Garnier, «La lecture de l'image médiévale », Le Livre au Moyen Âge, sous la dir. de Jean Glénisson, Paris, Éd. du CNRS, 1988, pp. 176-179.

49. Paris, Bibl. Arsenal, ms 5080, France, $X I^{e}$ siècle.

50. Par exemple Paris, BNF, $\mathrm{ms}$ fr 1136 fol. $33^{\mathrm{r}}$, France, $\mathrm{XIV}^{\mathrm{e}}$ siècle, reproduit dans $\mathrm{P}$. Riché et D. Alexandre-Bidon, op. cit., p. 203.

51. Par exemple, Paris, BNF, ms fr 1216 fol. $76^{\mathrm{r}}$. 
52. Paris, BNF, ms 580 fol. $57^{\mathrm{r}}$, reproduit dans P. Riché et D. Alexandre-Bidon, op. cit., p. 99.

53. Londres, British Libr., ms Egerton 3127 fol. $1^{\mathrm{v}}$.

54. Paris, BNF, ms fr 6185 fol. $143^{r}$.

55. Paris, BNF, ms fr 2000 fol. $50^{r}$.

56. Paris, BNF, ms fr 2829 notamment fol. $6^{\mathrm{r}}$.

57. Cantigas de santa Maria, fol. $228^{\mathrm{v}}$.

58. Suida-Manning coll.

59. Coll. part.

60. Vers 1330. Sienne, église San Agostino Novello.

61. Au fol. $228^{v}$.

62. On remarquera que dans le couple c'est encore le père, en l'occurrence Charles $V$, qui joue ce rôle lors d'une prière pour obtenir un enfant, en offrant à bout de bras un ex-voto au Christ : Paris, BNF, ms fr 2813 fol. $223^{\mathrm{r}}$. Reproduit dans P. Riché et D.

Alexandre-Bidon, op. cit., p. 39.

63. Voir la reproduction dans A. Martindale, «The Child in the Picture : a Medieval Perspective", The Church and the Child, D. Wood (ed.), Oxford, 1994, pp. 197-232 : p. 210.

64. Aux fol. $224^{\mathrm{r}}$ et $225^{\mathrm{r}}$.

65. C'est toujours le rôle du père ou d'un homme présent sur place : dans les Cantigas, dans les miracles de saint Louis illustrés au XIV et à la fin du XV siècle (Paris, BNF, ms fr 2829 et fr 5716 fol. $28^{\text {r }}$, reproduit dans P. Riché et D. Alexandre-Bidon, op. cit., p. 171), où l'on sauve une petite fille tombée dans le bief d'un moulin, ou encore dans un exvoto peint par Sano di Pietro où, pour une fois, c'est la mère qui prie tandis que l'homme pénètre dans la rivière pour en sortir le petit corps. Coll. part.

66. Autres exemples encore dans les Cantigas, notamment fol. $63^{\mathrm{r}}$.

67. C'est sans doute la raison pour laquelle saint Eustache, voyant ses deux enfants enlevés par des animaux sauvages qui s'enfuient dans deux directions opposées, ne cherche pas à courir après l'un et "se contente" de joindre les mains et prier : Lyon, BM, ms 867 , France, XIV ${ }^{\mathrm{e}}$ siècle.

68. Manuscrit exécuté en Syrie ou à Jérusalem, conservé au XIV siècle à Venise. Aujourd'hui à Vienne, Öst. Nl. Bibl., Cod. gr. theol. 31 fol. $30^{\text {r }}$.

69. Voir notamment le vif succès de tels motifs dans la gravure des Écoles du nord, dans la seconde moitié du XV siècle : bébés nus jouant avec ou sans leurs parents, tout seuls ou avec d'autres bébés, familles embrassées, etc., œuvres qui ont toutes chances d'avoir été exécutées par des artistes pères de famille!

70. Paris, BNF, ms fr 2829 fol. $64^{\mathrm{v}}$, reproduit dans P. Riché et D. Alexandre-Bidon, op. cit., p. 109.

71. Padoue, Salone. Image reproduite dans B. Laurioux, Le Moyen Âge à table, Paris, Adam Biro, 1989, p. 94.

72. Par exemple Paris, BNF, ms fr 6185 fol. $193^{\mathrm{v}}$.

73. Par exemple Cambridge, Fitzwilliam Museum, livre d'heures, vers 1500.

74. Paris, BNF, ms lat 9333 fol. $97^{\mathrm{v}}$ ou Vienne, Öst. Nl. Bibl., cod. Nova Series Vindobonensis 2644.

75. Par exemple, fol. $195^{\mathrm{r}}$.

76. Baltimore, ms 10290 fol. 69 .

77. Maître du Cabinet d'Amsterdam, «La sainte famille au rosier ». 
78. Reproduit dans P. Riché et D. Alexandre-Bidon, L'Enfant au Moyen Âge, Paris, Seuil/ BN, 1994, p. 15.

79. Paris, BNF, ms fr 22500.

80. New York, Metropolitan Museum of Art, 49. 7. 7. Reproduit dans P. Riché et D. Alexandre-Bidon, op. cit., p. 92.

81. Par exemple Martin Schongauer, 2e moitié du XV siècle. Paris, BNF, Département des Estampes.

82. Par exemple, Lyon, BM, ms 418 fol. $90^{\mathrm{v}}$.

83. Lyon, BM, ms PA 57 fol. $68^{\mathrm{V}}$ ou Paris, Bibl. Arsenal, ms 5070 (Décaméron, $\mathrm{VI}^{\mathrm{e}}$ journouvelle 10).

84. Chantilly, Musée Condé, ms 9 olim 1685 fol. 18v

85. Paris, BNF, ms fr 135 fol. $193^{\mathrm{r}}$, reproduit dans P. Riché et D. Alexandre-Bidon, op. cit., p. 32 .

86. Sur cette image voir D. Alexandre-Bidon, «Libertés et contraintes dans l'éducation des jeunes enfants à la fin du Moyen Âge ", Les Libertés au Moyen Âge, Montbrison, 1987, pp. 241-252.

87. Par exemple Paris, BNF, ms fr $9198-99$ fol. $68^{\mathrm{r}}$, Miracles de Notre Dame, Flandre, milieu du XVe siècle ou Copenhague, Kgl Bibl. Thott, 574.4 fol. 13v, Livre d'heures, Angleterre, fin du XIVe siècle.

88. La Haggadah de Sarajevo, Paris, Éd. Siloé, s. d., n. p.

89. I. G. Marcus, Rituals of Childhood. Jewish Acculturation in Medieval Europe, Yale University Press, New Haven and London, 1996.

90. T. et M. Metzger, La Vie juive au Moyen Âge illustrée par les manuscrits hébraïques enluminés du XIII ${ }^{e}$ au XVI e siècle, Office du Livre, Vilo, 1982., reproduit p. 114.

91. Je remercie le Pr. Joseph Shatzmiller, qui m'a aimablement communiqué sa traduction anglaise de ce texte.

92. Paris, BNF, ms fr 2829 fol. $98^{\vee}$, reproduit dans P. Riché et D. Alexandre-Bidon, op. cit., p. 176.

93. Paris, BNF, ms fr 3115 fol. $104^{\mathrm{r}}$.

94. Jérusalem, Musée d'Israël, ms 180/51 fol. 64v .

95. Paris, BNF, ms fr 202 fol. $15^{\mathrm{v}}$, reproduit dans P. Riché et D. Alexandre-Bidon, op. cit., p. 91.

96. Stéréotypée ou non, cette vision sexuée du monde a tout de même eu des répercussions sociales indéniables, notamment dans le domaine de l'enseignement : ainsi les garçons avaient-ils, au Moyen Âge, des maîtres d'école et les filles des maîtresses.

97. Paris, BNF, ms lat 8504. Avant 1313.

98. Par exemple, Éthiques d'Aristote, Bruxelles, Bibl. Roy. Albert $\mathrm{I}^{\mathrm{er}}$, ms $9505-9506$ fol. $2^{\mathrm{v}}$.

99. Paris, BNF, ms fr 473 fol. $1^{\mathrm{r}}$. 1374.

100. Par exemple, Liverpool Museum, ms M 12001 fol. $237^{\mathrm{v}}$.

101. Madrid, Archivo Historico Nacional, cod. $\mathrm{n}^{\circ} 1002$.

102. New York, Pierpont Morgan Libr., ms 729 fol. $1^{v}$, reproduit dans P. Riché et D. Alexandre-Bidon, op. cit., p. 100.

103. Décaméron, Paris, Bibl. Arsenal, ms 5070.

104. Une exception dans les Heures de la duchesse de Savoie, où adultes des deux sexes jouent aux boules de neige avec des petits garçons : Chantilly, musée Condé, ms 76 fol. 12v , reproduit dans P. Riché et D. Alexandre-Bidon, op. cit., p. 196. 
105. D'après un dessin de Benedetto da Milano, vers 1500, mois de novembre et décembre.

106. Paris, BNF, ms fr 5054 fol. $195^{\mathrm{v}}$.

107. Apocalypse de Jean, Paris, BNF, ms fr 13096 fol. $60^{\mathrm{v}}$, reproduit dans P. Riché et D. Alexandre-Bidon, op. cit., p. 20.

108. Paris, Bibl. de l'Arsenal, ms 5219 fol. $93^{\mathrm{r}}$.

109. Paris, BNF, ms fr 15397 fol. 32v

\section{AUTEUR}

DANIÈLE ALEXANDRE-BIDON

EHESS, CIHAM/Lyon II - UMR 5648 